\title{
A new experimental approach to the search for chemical density factors in the regulation of monoculture growth
}

\author{
Andrei G. Degermendzhy, ${ }^{*}$ Vera V. Adamovich and Vladimir A. Adamovich \\ Laboratory of Biophysics of Ecosystems, Institute of Biophysics, Siberian Branch of the Russian Academy of Sciences, \\ 660036 Krasnoyarsk, Russia
}

(Received 1 April 1992; revised 5 February 1993; accepted 25 February 1993)

\begin{abstract}
In monocultures of micro-organisms, growth is controlled by feedback mechanisms involving chemical factors such as limiting substrates and inhibitory metabolic products. The role of such feedback in the growth regulation of Escherichia coli 0-124 was investigated by growing cells in batch culture using a medium containing glucose and mineral salts. In various phases of growth, portions of the native culture were diluted with culture filtrate, so that although cell density decreased, the chemical composition of the growth medium was unaltered. As the diluted cultures grew, variations in growth acceleration were calculated and compared with those of native (undiluted) cultures. Towards the end of the exponential phase and in the growth deceleration phase, the specific feedback level (FBL) was between -20 and $-200\left(\mathrm{~h} \mathrm{~g} \mathrm{l}^{-1}\right)^{-1}$. The feedback components resulting from changes in glucose concentration were calculated using experimentally determined values of $\mu_{\max }\left(0.55 \pm 0.05 \mathrm{~h}^{-1}\right)$ and $K_{\mathrm{s}}$ $\left(2.5 \pm 0.7 \mathrm{mg} \mathrm{l}^{-1}\right)$. Only $0 \cdot 1-40 \%$ of FBL could be accounted for by changes in glucose concentration, indicating the presence of additional growth regulators. The method developed may become a new tool for determination of growth-regulating cell-density factors in microbial cultures.
\end{abstract}

\section{Introduction}

The study of regulation of microbial population growth is an important aspect of population ecology. Knowledge of growth-limitation mechanisms, chemical regulators and the dependence of the specific growth rate $(\mu)$ on environmental factors are equally important for bioengineering, biological systems for sewage treatment, modelling water purification processes, predicting water quality and understanding the dynamics of aquatic ecosystems, etc. The most important chemical substances in this context are those which provide stability of microbial populations. These are primarily growth control density factors (GCDFs), i.e. substances excreted or consumed by the population and affecting its growth (Fredrickson, 1977; Fredrickson \& Stephanopoulos, 1981). The dependence of $\mu$ on a particular GCDF (as in the Michaelis-Menten relationship, for example) may be determined experimentally, but usually it is not known

*Author for correspondence. Tel. 01173912452722 ; fax 0117391 2 222556; e-mail ibp@ibp.krasnoyarsk.su.

Abbreviations: FBL, feedback level; GCDF, growth control density factor. whether $\mu$ is also affected by other unknown GCDF(s). Is it, therefore, possible to estimate quantitatively the growth regulation of a particular species in a given system, if the $\mu$-GCDF relationship is known?

An approach to the solution of this problem is as follows. Let us consider a particular population, which is a monoculture in which growth is controlled by feedback mechanisms involving several chemical GCDFs. The GCDFs may be represented not only by limiting substrates but also by metabolic products inhibiting or stimulating growth (Smith, 1975; Slyter \& Weaver, 1977). In this case, what is an integral measure of the feedback level (FBL) in growth regulation, or an overall estimate of the effect of all GCDFs on population growth? Our theory suggests that this is the difference between the growth accelerations $(B)$ of experimental (perturbed) and native (unperturbed) cultures which is observed when the population concentration $(\Delta X)$ is altered, while the chemical composition of the medium remains unchanged (Degermendzhy et al., 1989):

$$
B=\left.\frac{\partial \mu}{\partial t}\right|^{p}-\left.\frac{\partial \mu}{\partial t}\right|^{u}
$$

where $p$ and $u$ represent perturbed and unperturbed cultures respectively. 
In the general case of monoculture, in which $\mu$ is determined by $n$ GCDFs, the equation for $B\left(\equiv B_{t}\right)$ normalized to the unit of variation of biomass concentration and the unit of $\mu$ takes the form:

$$
B_{t}=\sum_{i=1}^{n}\left(\frac{\partial \mu}{\partial A_{i}}\right) a_{i}
$$

where $\mu\left(A_{1}, A_{2}, \ldots A_{n}\right)$ is the specific growth rate of the monoculture as a function of GCDF, $A_{1}, A_{2}, \ldots A_{n} ; a_{i}$ is the coefficient of excretion $\left(a_{i}>0\right)$ or consumption $\left(a_{i}<0\right)$ of the $i$ th GCDF as the biomass concentration is changed by unity; and $\partial \mu / \partial A_{i}$ is the partial derivative of $\mu$ with respect to the $i$ th factor.

Thus, all $n$ GCDFs make a certain contribution $\left(a_{i} \cdot \partial \mu / \partial A_{i}\right)$ to the integral theoretical value of $B_{i}$. On the other hand, a value equivalent to $B_{t}$ can be found experimentally $\left(B_{\mathrm{e}}\right)$ from the above verbal definition of FBL (as a response in the form of growth acceleration or deceleration found by comparison of the dynamics of biomass of the experimental and the native populations). If only one GCDF (let it be marked by 1) and its characteristics $\left(\hat{\partial} \mu / \partial A_{1}\right.$ and $\left.a_{1}\right)$ are known, the difference

$$
\Delta \equiv B_{\mathrm{e}}-\frac{\partial \mu}{\partial A_{1}} \cdot a_{1}=\sum_{i=2}^{n} \frac{\partial \mu}{\partial A_{i}} a_{i}
$$

will determine the total contribution of other unknown GCDFs. Thus, if the difference is equal to zero $(\Delta=0)$, only one GCDF (marked by 1) takes part in regulation [there is also a low probability of strict equality between positive and negative feedbacks for the factors $i=2, \ldots n$ (i.e. $\Delta=0$ )]. If not, the existence of other GCDFs is possible. In this case, one can estimate the percentage contribution to regulation of all the remaining GCDFs as $\left(\Delta / B_{\mathrm{e}}\right) 100 \%$. This is an important value, because it shows the magnitude of the integral effect on regulation of an unknown GCDF. If the value is low $(<10 \%)$, a search for GCDF(s) is obviously not expedient. If, however, values are higher, the search for other GCDFs should be continued.

In this work we sought to estimate FBL experimentally, for monocultures of Escherichia coli growing in a glucose medium, and to determine the contribution to FBL of glucose as the known GCDF.

\section{Methods}

Organism and medium. Escherichia coli $\mathrm{O}-124$ (isolated and identified by the Krasnoyarsk Epidemiological Station) was grown in a synthetic mineral medium of the following composition $\left(\mathrm{g} \mathrm{l}^{-1}\right): \mathrm{Na}_{2} \mathrm{HPO}_{4}(6)$, $\mathrm{KH}_{2} \mathrm{PO}_{4}(3), \mathrm{NaCl}(5), \mathrm{NH}_{4} \mathrm{Cl}(1), \mathrm{MgSO}_{4}\left(1 \mathrm{ml} \mathrm{l} \mathrm{l}^{-1}\right.$ of a $1 \mathrm{M}$ solution), $\mathrm{CaCl}_{2}\left(1 \mathrm{ml} \mathrm{l}^{-1}\right.$ of a $0.1 \mathrm{~m}$ solution); $\mathrm{pH} 6 \cdot 7-7 \cdot 0$. Glucose, at various concentrations, was used as a source of carbon and energy. The medium was autoclaved for $0.5 \mathrm{~h}$ at $1 \mathrm{~atm}$.

Growth conditions. E. coli, maintained on slopes of a nutrient agar medium (Kuznetsov \& Dubinina, 1989), was transferred to liquid medium ( $1 \mathrm{~g}$ glucose $\left.\mathrm{I}^{-1}\right)$ and incubated at $26^{\circ} \mathrm{C}$ on a shaker until a dry biomass concentration of $0.1-0.2 \mathrm{~g} \mathrm{t}^{-1}$ was reached. These cultures were then inoculated into Erlenmeyer flasks ( $500 \mathrm{ml}$ ) containing $200 \mathrm{ml}$ fresh medium, with various glucose concentrations, and were similarly incubated.

Measure of culture optical density, cell concentration and biomass. E. coli biomass was monitored throughout the entire period of cultivation. Cell concentration was determined by direct microscopic analysis on membrane filters (pore diameter $0.2 \mu \mathrm{m}$ ) at $1000 \times$ magnification using Razumov's technique (Kuznetsov \& Dubinina, 1989) and phenol erythrosin.

To determine biomass concentration, $10-20 \mathrm{ml}$ of the culture was filtered through a boiled Synpor N7 filter (pore diameter $0.16 \mu \mathrm{m}$ ). The filters were washed in $\mathrm{HCl}$ solution $\left(1 \mathrm{ml}\right.$ conc. $\left.\mathrm{HCl}^{-1}\right)$ and dried at $80^{\circ} \mathrm{C}$ for $1 \mathrm{~h}$. Weights of the filters before and after filtering were determined to an accuracy of $0.15 \mathrm{mg}$.

Culture optical density was determined at $540 \mathrm{~nm}$. The relationship between dry weight $(X)$ and culture optical density $\left(X_{\mathrm{OD}}\right)$ was linear $\left[X=C \cdot X_{\mathrm{OD}}+b\right.$, where values of the constants (with errors) were: $C=0.22 \pm 0.007 \mathrm{~g} \mathrm{I}^{-1}$ per $\mathrm{OD}_{540}$ unit and $\left.b=-0.004 \pm 0.003\right]$.

Determination of the yield coefficient and residual glucose concentration. The yield coefficient for $E$. coli on glucose was calculated from the linear regression of initial glucose concentration $\left(S_{0}\right)$ and maximum biomass concentration $\left(X_{\max }\right)$ in batch culture $\left[X_{\max }=\right.$ $Y \cdot S_{0}+v$, where the yield coefficient was $Y=0.325 \pm 0.015 \mathrm{~g}$ (g glucose) $^{-1}$ and $v$ (a constant) $\left.=0.004 \pm 0.002 \mathrm{~g} \mathrm{l}^{-1}\right]$. The same relation was used for calculation of the residual glucose concentration $(S)$ at any moment of cultivation.

Determination of the dependence of $\mu$ on glucose concentration (S). The dependence of $\mu$ on glucose concentration was determined using media with various initial glucose concentrations $\left(0-500 \mathrm{mg} \mathrm{l}^{-1}\right)$. $\mu$ was calculated by the equation:

$$
\mu=\ln \left(X_{t}-\ln X_{0}\right) / \Delta t
$$

where $X_{0}$ and $X_{t}$ are the initial and final biomass concentrations, and $\Delta t$ is the incubation time. Cell number was used to estimate biomass at $S_{0}<10 \mathrm{mg} \mathrm{l}^{-1}$; at $S_{0}>10 \mathrm{mg} \mathrm{l}^{-1}, \mathrm{OD}_{540}$ was used. The incubation

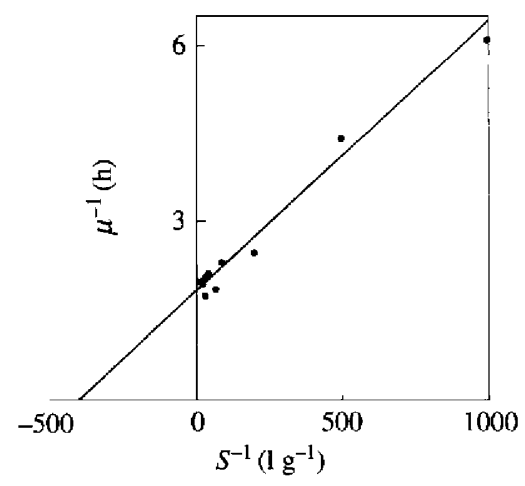

Fig. 1. Reciprocal plot of the dependence of the specific growth rate of E. coli $\left(\mu^{-1}\right)$ versus glucose concentration $\left(S^{-1}\right)$. The intercepts give $\mu_{\max }$ $=0.55 \mathrm{~h}^{-1}, K_{\mathrm{s}}=2.5 \mathrm{mg} \mathrm{l}^{-1}$. 
Table 1. Change in $O D_{540}$ of $E$. coli $O-124$ in experiment $1 S_{0}=500 \mathrm{mg} \mathrm{l}^{-1}$ )

For notation see Fig. 2 and the text.

\begin{tabular}{ccccccc}
\hline \hline $\begin{array}{c}S \\
\left(\mathrm{mg} \mathrm{l}^{-1}\right)\end{array}$ & $\begin{array}{c}\Delta t \\
(\mathrm{~h})\end{array}$ & $U_{-1}$ & $U_{0}$ & $U_{1}$ & $P_{0}$ & $P_{1}$ \\
\hline 175 & 0.28 & 0.408 & 0.432 & 0.458 & 0.225 & 0.252 \\
162 & 0.25 & 0.428 & 0.450 & 0.482 & 0.210 & 0.238 \\
150 & 0.33 & 0.435 & 0.466 & 0.530 & 0.254 & 0.297 \\
132 & 0.23 & 0.457 & 0.490 & 0.538 & 0.254 & 0.272 \\
109 & 0.30 & 0.466 & 0.520 & 0.584 & 0.270 & 0.300 \\
79 & 0.18 & 0.520 & 0.560 & 0.600 & 0.310 & 0.330 \\
32 & 0.18 & 0.592 & 0.622 & 0.652 & 0.347 & 0.374 \\
13 & 0.25 & 0.610 & 0.648 & 0.666 & 0.361 & 0.373 \\
5 & 0.18 & 0.646 & 0.662 & 0.665 & 0.362 & 0.370 \\
\hline \hline
\end{tabular}

times chosen were short $(1-2 h)$; thus glucose concentration did not significantly decrease.

The dependence of $\mu$ on glucose concentration was approximated by the Michaelis-Menten equation (Fig. 1). The constants $\mu_{\max }$ and $K_{5}$, determined by nonlinear regression (Pinto \& Oestreicher, 1983) were: $\mu_{\max }=0.55 \pm 0.05 \mathrm{~h}^{-1}, K_{\mathrm{s}}=2.5 \pm 0.7 \mathrm{mg} \mathrm{l}^{-1}$

Determination of FBL. FBLs of E. coli were experimentally measured as follows. Portions (up to $50 \mathrm{ml}$ ) of the native cultures, grown on media with initial glucose concentrations from 300 to $500 \mathrm{mg} \mathrm{l}^{-1}$, were taken in various phases of growth and filtered through a bacterial filter (Vladipor $\mathrm{N} 2$; pore diameter $0.2 \mu \mathrm{m}$ ), previously boiled in distilled water. Then the filtrate and part of the native culture were mixed in various proportions in separate sterile flasks to create cultures with decreased $E$. coli concentrations. Both experimental and reference (native) cultures were incubated on a shaker at $26^{\circ} \mathrm{C}$ and changes in biomass concentration were determined by $\mathrm{OD}_{540}$ measurements. Methodological difficulties prevented the replication of each individual experimental run more than once. Thus, errors presented in this paper are derived from estimates of the errors involved in the various measurements made.

The methods of calculation of FBL and the contribution of GCDF to FBL are shown in the Appendix

\section{Results and Discussion}

\section{Determination of feedback level}

The FBL of E. coli, growing in batch culture and in various growth phases, was determined in six experiments. In the technique used, bacterial concentration was reduced by diluting cultures with culture filtrate. Thus, cells trapped on filters, which might change their physiological state after filtration, were not introduced into the experimental culture. It was not determined whether filtration affected the composition of the filtered medium.

Results for one experiment are listed in Table 1, and Table 2 shows the calculation of experimental $\left(B_{\mathrm{e}}\right)$ and theoretical $\left(B_{t}\right)$ values for FBL. Theoretical FBL values were calculated on the assumption that glucose was the only factor which controlled growth rate, and were based on the values of $Y, \mu_{\max }$ and $K_{\mathrm{s}}$ determined in this study (see Methods and Appendix).

Table 2. Theoretical $\left(B_{i}\right)$ and experimental $\left(B_{e}\right)$ values for $F B L$ with errors $(\Delta B)$

For notation see the text.

\begin{tabular}{|c|c|c|c|c|c|c|c|c|}
\hline $\begin{array}{c}S_{0} \\
\left(\mathrm{~g} \mathrm{l}^{-1}\right)\end{array}$ & $\begin{array}{c}\text { Expt } \\
\text { no. }\end{array}$ & $\begin{array}{c}S \pm \Delta S \\
\left(\mathrm{~g} \mathrm{l}^{-1}\right)\end{array}$ & $k$ & $B_{\mathrm{e}}^{*}$ & $\begin{array}{c}\Delta B_{\mathrm{e}} / B_{\mathrm{e}} \\
(\%)\end{array}$ & $B_{t}^{*}$ & $\begin{array}{c}\Delta B_{t} / B_{t} \\
(\%)\end{array}$ & $\begin{array}{c}B_{t} / B_{e} \\
(\%)\end{array}$ \\
\hline \multirow[t]{2}{*}{$0 \cdot 3$} & 1 & $\begin{array}{r}0.07 \pm 0.010 \\
0.045 \pm 0.005\end{array}$ & $\begin{array}{l}0.50 \\
0.50\end{array}$ & $\begin{array}{r}-140 \\
-38\end{array}$ & $\begin{array}{l}57 \\
36\end{array}$ & $\begin{array}{l}-0.79 \\
-1.80\end{array}$ & $\begin{array}{l}75 \\
80\end{array}$ & $\begin{array}{l}0 \cdot 56 \\
4 \cdot 74\end{array}$ \\
\hline & 2 & $\begin{array}{l}0.065 \pm 0.010 \\
0.065 \pm 0.010 \\
0.065 \pm 0.010\end{array}$ & $\begin{array}{l}0.38 \\
0.56 \\
0.67\end{array}$ & $\begin{array}{l}-26 \\
-37 \\
-75\end{array}$ & $\begin{array}{l}56 \\
45 \\
29\end{array}$ & $\begin{array}{l}-0.92 \\
-0.92 \\
-0.92\end{array}$ & $\begin{array}{l}81 \\
81 \\
81\end{array}$ & $\begin{array}{l}3 \cdot 54 \\
2 \cdot 49 \\
1 \cdot 22\end{array}$ \\
\hline \multirow[t]{4}{*}{0.5} & 1 & $\begin{array}{r}0.175 \pm 0.010 \\
0 \cdot 162 \pm 0.010 \\
0.15 \pm 0.010 \\
0.032 \pm 0.005 \\
0.005 \pm 0.001\end{array}$ & $\begin{array}{l}0.52 \\
0.47 \\
0.54 \\
0.56 \\
0.54\end{array}$ & $\begin{array}{r}-150 \\
-140 \\
-35 \\
-110 \\
-220\end{array}$ & $\begin{array}{l}49 \\
49 \\
55 \\
38 \\
59\end{array}$ & $\begin{array}{l}-0.134 \\
-0 \cdot 156 \\
-0 \cdot 182 \\
-3 \cdot 55 \\
-74\end{array}$ & $\begin{array}{l}63 \\
65 \\
65 \\
76 \\
70\end{array}$ & $\begin{array}{r}0.09 \\
0.11 \\
0.52 \\
3.23 \\
33.65\end{array}$ \\
\hline & 2 & $\begin{array}{l}0.018 \pm 0.003 \\
0.013 \pm 0.002\end{array}$ & $\begin{array}{l}0.25 \\
0 \cdot 41\end{array}$ & $\begin{array}{r}-170 \\
-41\end{array}$ & $\begin{array}{l}33 \\
71\end{array}$ & $\begin{array}{l}-10 \\
-17\end{array}$ & $\begin{array}{l}73 \\
65\end{array}$ & $\begin{array}{r}5 \cdot 88 \\
41 \cdot 46\end{array}$ \\
\hline & 3 & $\begin{array}{r}0 \cdot 160 \pm 0 \cdot 010 \\
0 \cdot 139 \pm 0 \cdot 010 \\
0 \cdot 120 \pm 0.010 \\
0 \cdot 118 \pm 0 \cdot 010 \\
0 \cdot 10 \pm 0 \cdot 010 \\
0 \cdot 041 \pm 0.005\end{array}$ & $\begin{array}{l}0.46 \\
0.55 \\
0.22 \\
0.38 \\
0.58 \\
0.51\end{array}$ & $\begin{array}{l}-28 \\
-24 \\
-60 \\
-67 \\
-62 \\
-22\end{array}$ & $\begin{array}{l}60 \\
78 \\
45 \\
30 \\
56 \\
90\end{array}$ & $\begin{array}{l}-0.16 \\
-0.21 \\
-0.27 \\
-0.29 \\
-0.41 \\
-2.45\end{array}$ & $\begin{array}{l}62 \\
66 \\
66 \\
69 \\
70 \\
73\end{array}$ & $\begin{array}{r}0.57 \\
0.88 \\
0.45 \\
0.43 \\
0.66 \\
11.14\end{array}$ \\
\hline & 4 & $\begin{array}{r}0.15 \pm 0.010 \\
0.087 \pm 0.012\end{array}$ & $\begin{array}{l}0.47 \\
0.49\end{array}$ & $\begin{array}{l}-32 \\
-75\end{array}$ & $\begin{array}{l}41 \\
37\end{array}$ & $\begin{array}{l}-0.18 \\
-0.79\end{array}$ & $\begin{array}{l}65 \\
75\end{array}$ & $\begin{array}{l}0.56 \\
1.05\end{array}$ \\
\hline
\end{tabular}

${ }^{*} B_{\mathrm{e}}$ and $B_{\mathrm{t}}$ are expressed as $\left(\mathrm{h} \mathrm{g}^{-1}\right)^{-1}$. 
Table 2 shows only those experimental values of FBL with relative errors less than $100 \%$. Almost half of the FBL values determined had errors exceeding $100 \%$. High errors were found particularly at the beginning of the exponential phase of growth; however, the absolute values of FBL in these cases did not exceed $10\left(\mathrm{~h} \mathrm{~g}^{-1}\right)^{-1}$. To calculate $B_{\mathrm{e}}$ values, we used the approximation of equation (9) (see Appendix) to determine values of $\mu$ (i.e. $\left.\mu_{0}\right)$ at the moment of culture dilution. We estimate that this may introduce a further error of up to $10-30 \%$.

The results in Table 2 show a significant difference between experimental and theoretical FBL values at the end of the exponential growth phase and in the phase of growth deceleration. This is evidence for the presence of important regulators other than glucose concentration in the system. The percentage of FBL attributable to glucose regulation $\left[\left(B_{\mathrm{t}} / B_{\mathrm{e}}\right) \times 100 \%\right.$, Table 2] ranged from 0.1 to $40 \%$ in the various experiments. Thus, other unknown GCDFs contributed about $60-99.9 \%$ of the total FBL.

The disadvantage of the method developed here for determining FBL is the high calculated error, which is a common defect of analyses where differential factors are involved. This drawback might be avoided by using alternative methods to determine the increase in the biomass concentration, for example by measuring the rate of uptake of labelled substrates, or the rate of oxygen consumption (for aerobic organisms). In these cases the first derivative of the measurements made (not the second as required in the analysis used in this paper) will enable variations in growth acceleration to be estimated, thus improving accuracy of FBL determinations. Chemostat continuous culture techniques may also be applied to the determination of GCDFs. The major advantage of continuous cultivation over batch cultivation is that the former can be maintained in a steady state. This will enable more accurate evaluation of growth parameters $\left(\mu_{\max }, K_{s}, S\right)$, and of FBL values at low concentrations of the limiting substrate. This approach is currently under investigation.

The experimental results obtained here lead us to believe that the technique developed will become a useful microbiological tool for evaluating the role of chemical regulators of monoculture growth. Other theoretically possible means of determining GCDF, that have not yet been realized experimentally, are described in a previous paper (Degermendzhy et al., 1989).

\section{Appendix}

\section{Calculation of $F B L$ and its errors}

Let the experimental results for FBL be as represented in Fig. 2. Generally, the dynamics of biomass concen- tration, $X$, in batch culture is described by the differential equation:

$$
\frac{\mathrm{d} X}{\mathrm{~d} t}=\mu(t) \cdot X
$$

where $\mu(t)$ is the specific growth rate $\left(\mathrm{h}^{-1}\right)$. If the time interval $\left[t_{0}, t_{1}\right]$ is infinitesimally small $(\Delta t)$, then:

$$
\mu(t)=\mu_{0}+\alpha \cdot \Delta t
$$

The biomass concentration dynamics (3) with a correction for (4) takes the form:

$$
\ln \left(X / X_{0}\right)=\mu_{0} \cdot \Delta t+\alpha \cdot \Delta t^{2} / 2
$$

where $X_{0}$ is the biomass concentration at time $t_{0}$. Thus, the growth acceleration value, $\alpha$, is determined from (5) as:

$$
\alpha=2\left[\ln \left(X / X_{0}\right)-\mu_{0} \Delta t\right] / \Delta t^{2}
$$

Assume that $\alpha$ is the growth acceleration corresponding to the unperturbed dynamics (curve I, Fig. 2) and $\beta$ is the one that corresponds to the perturbed dynamics (curve II). Thus, the difference,

$$
\beta-\alpha=\left.\frac{\partial \mu}{\partial t}\right|_{\mathrm{II}} ^{t_{0}}-\left.\frac{\partial \mu}{\partial t}\right|_{\mathrm{I}} ^{t_{0}}=B_{\mathrm{e}}^{\text {total }}
$$

is the experimental total FBL in self-regulation of the
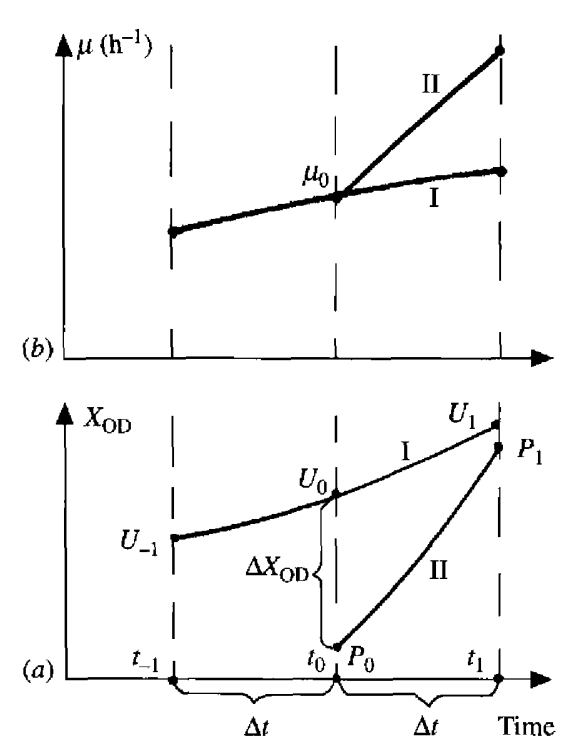

Fig. 2. (a) Speculative dynamics of optical density $\left(X_{\mathrm{OD}}\right)$ for perturbed (diluted) and unperturbed cultures (curves II and I respectively). $U_{-1}$, $U_{0}, U_{1}$ are the unperturbed values at the time moments $t_{-1}, t_{0}, t_{1} . P_{0}, P_{1}$ are the values at times $t_{0}, t_{1}$ after dilution of the monoculture $\left(\Delta X_{\mathrm{OD}}=\right.$ $\left.P_{0}-U_{0}\right)$ at time $t_{0} ; k=P_{0} / U_{0}$ is the coefficient of dilution. (b) Speculative dynamics of $\mu$ for unperturbed (curve I) and perturbed (curve II) cultures. Notations are as in the text. 
monoculture growth $(\beta$ and $\alpha$ are tangents of curve II and curve I, respectively). Substituting from equation (6) and using the notation of Fig. 2,

$$
B_{\mathrm{e}}^{\text {total }}=\frac{2}{\Delta t^{2}} \cdot \ln \left(\frac{P_{1} U_{0}}{P_{0} U_{1}}\right)
$$

Since $B_{\mathrm{e}}^{\text {total }}$ depends on the magnitude of the dilution $(\Delta X)$ and on $\mu$, we will consider a specific (normalized) value of the FBL:

$$
B_{\mathrm{e}}=\frac{B_{\mathrm{e}}^{\text {total }}}{\Delta X \cdot \mu_{0}}=\frac{4}{\Delta t} \cdot \ln \left(\frac{P_{1} U_{0}}{P_{0} U_{1}}\right) /\left(\frac{P_{0}}{U_{0}}-1\right) \cdot C\left(U_{1}-U_{-1}\right)
$$

where $\Delta X=P_{0}-U_{0}$ is the change in biomass concentration due to dilution. In equation (8) the value of $\mu_{0}$, the specific growth rate at the moment $t_{0}$, is calculated by the Lagrange equation:

$$
\mu_{0}=(\mathrm{d} X / \mathrm{d} t) / X \approx\left(U_{1}-U_{-1}\right) / 2 \cdot \Delta t \cdot U_{0}
$$

The absolute error $\left(\Delta B_{\mathrm{e}}\right)$ of $B_{\mathrm{e}}$ takes the form:

$$
\begin{array}{r}
\Delta B_{\mathrm{e}}=\left|B_{\mathrm{e}}\right| \cdot\left[\frac{d\left(\frac{1}{P_{1}}+\frac{1}{U_{0}}+\frac{1}{P_{0}}+\frac{1}{U_{1}}\right)}{\left|\ln \left(\frac{P_{1}}{k U_{1}}\right)\right|}+\frac{d\left(\frac{1}{U_{0}}+\frac{k}{U_{0}}\right)}{|k-1|}\right. \\
\left.+\frac{\Delta C\left|U_{1}-U_{-1}\right|+2 C d}{C\left|U_{1}-U_{-1}\right|}\right]
\end{array}
$$

and may be estimated if the values $d, C, \Delta C$ are known. The error in determining optical density, $d$, was assumed to be the same for all measurements and was equal to 0.001 OD units; $\Delta C=0.007$ (see Methods) $; \mathrm{k}=P_{0} / U_{0}$.

\section{Calculation of the contribution of known GCDF to total regulation}

Let $\mu(S)=\mu_{\max } S /\left(K_{\mathrm{s}}+S\right)$ be the dependence of specific growth rate on glucose concentration. Then the theor- etical dynamics of both biomass and substrate concentrations is described by the equations: $\dot{X}=\mu \cdot X$; $\dot{S}=-\mu \cdot X / Y$, where $Y$ is the yield coefficient $(\dot{X}=$ $\mathrm{d} X / \mathrm{d} t ; \dot{S}=\mathrm{d} S / \mathrm{d} t)$. Assuming that glucose concentration is the only regulator of growth rate, the theoretical specific coefficient of feedback, according to (2), will take the form:

$$
B_{\mathrm{t}}=-\frac{1}{\mathrm{Y}} \cdot \frac{\partial \mu}{\partial S}=\frac{-\mu_{\max } K_{\mathrm{s}}}{Y\left(K_{\mathrm{s}}+S\right)^{2}}
$$

The absolute error of the value of $B_{t}$ takes the form:

$$
\Delta B_{t}=\left|B_{t}\right| \cdot\left[\frac{\Delta \mu_{\max }}{\mu_{\max }}+\frac{\Delta Y}{Y}+\frac{\Delta K_{\mathrm{s}}\left|S-K_{\mathrm{s}}\right|}{K_{\mathrm{s}}\left(K_{\mathrm{s}}+S\right)}+\frac{2 \Delta S}{K_{\mathrm{s}}+S}\right]
$$

The value of the substrate concentration, at any time, is determined from the relationship $S=\left[S_{0}-(X / Y)\right]$ and its error is determined as:

$$
\Delta S=\frac{\Delta Y \cdot X}{Y^{2}}+\frac{C \cdot d}{Y}+\frac{X \Delta C}{Y \cdot C}
$$

This work was supported by Krasnoyarsk Regional Scientific Foundation grant 1F0185.

\section{References}

Degermendzhy, A. G., Adamovich, V. A. \& Pozdyaev, V. N. (1989). On the cybernetics of bacterial communities: observation, experiments and theory. Cybernetics and Systems 20, 501-540.

Fredrickson, A. G. (1977). Behavior of mixed cultures of microorganisms. Annual Review of Microbiology 31, 63-87.

Fredrickson, A. G., Stephanopoulos, G. (1981). Microbial competition. Science 213, 972-979.

KuzNETsov, S. I. \& Dubinina, G. A. (1989). Methods of Investigation of Aqueous Microorganisms (in Russian). Moscow: Nauka.

Pinto, G. E. \& Oestricher, E. G. (1983). Pocket computer program for fitting the Michaelis-Menten equation. Computation in Biology and Medicine 13, 309-315.

SLyter, L. L. \& WeAver, I. M. (1977). Tetrahydrofolate and other growth requirements of certain strains of Ruminococcus flavefaciens. Applied and Environmental Microbiology 33, 363-369.

SMITH, I. S. (1975). The nature of the stimulation of the growth of Streptococcus lactis by yeast extract. Journal of Dairy Research 42, $123-138$. 